\title{
siRNA Inhibition of Endocytic Pathways to Characterize the Cellular Uptake Mechanisms of Folate-Functionalized Glycol Chitosan Nanogels
}

Paula Pereira, ${ }^{\dagger}$ Sílvia S. Pedrosa, ${ }^{\dagger}$ Jennifer M. Wymant, ${ }^{\ddagger}$ Edward Sayers, ${ }^{\ddagger}$ Alexandra Correia, ${ }^{\S}$ Manuel Vilanova, ${ }^{\S}, \|$ Arwyn T. Jones, ${ }^{\ddagger}$ and Francisco M. Gama* ${ }^{, \dagger}$

${ }^{\dagger}$ Institute for Biotechnology and Bioengineering, Centre of Biological Engineering, Campus de Gualtar, University of Minho, Braga 4710-05, Portugal

${ }^{\ddagger}$ Cardiff School of Pharmacy and Pharmaceutical Sciences, Cardiff University, Redwood Building, Cardiff CF10 3NB, Wales

${ }^{\S}$ Institute of Molecular and Cell Biology, Rua Campo Alegre, Porto 4099-003, Portugal

"Abel Salazar Biomedical Sciences Institute, University of Porto, Rua de Jorge Viterbo Ferreira No. 228, Porto 4050-313, Portugal

Supporting Information

ABSTRACT: Glycol chitosan nanogels have been widely used in gene, drug, and contrast agent delivery in an effort to improve disease diagnosis and treatment. Herein, we evaluate the internalization mechanisms and intracellular fate of previously described glycol chitosan nanogels decorated with folate to target the folate receptor. Uptake of the folatedecorated nanogel was impaired by free folate, suggesting competitive inhibition and shared internalization mechanisms via the folate receptor. Nanogel uptake was shown to occur mainly through flotillin-1 and Cdc42-dependent endocytosis. This was determined by inhibition of uptake reduction

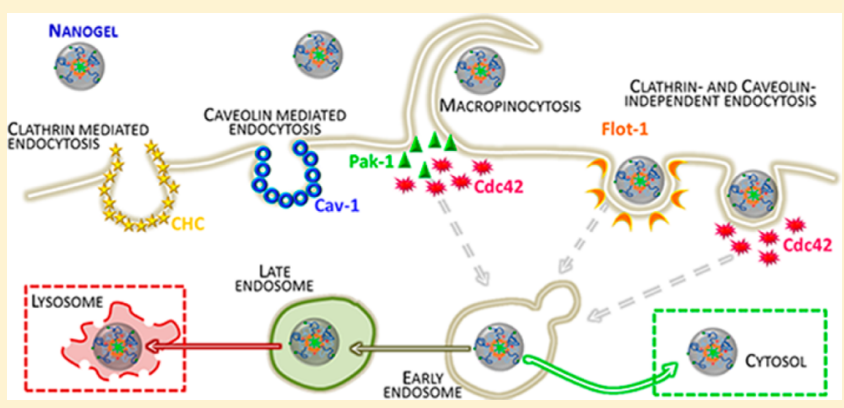
observed upon siRNA depletion of these two proteins and the pathways that they regulate. The data also suggest the involvement of the actin cytoskeleton in nanogel uptake via macropinocytosis. After $7 \mathrm{~h}$ of incubation with HeLa cells, approximately half of the nanogel population was localized in endolysosomal compartments, whereas the remaining $50 \%$ of the material was in undefined regions of the cytoplasm. Glycol chitosan nanogels may thus have potential as drug delivery vectors for targeting different intracellular compartments.

KEYWORDS: glycol chitosan nanogel, folate, siRNA transfection, endocytic pathways, intracellular localization

\section{INTRODUCTION}

Macromolecular micelles, commonly termed nanogels, have been synthesized, characterized, and studied for numerous biomedical applications including delivery of therapeutic entities. Active nanogel targeting strategies have been conceived to enhance their site-specific delivery by, for example, decorating the surface with ligands of plasma membrane receptors that are overexpressed on target cells. ${ }^{1}$

A major advantage in the use of nanoparticles as drug delivery systems lies in their amenability to modifications that allow them to cross biological barriers. The cell membrane is naturally impermeable to complexes larger than $1 \mathrm{kDa}$; however, nanoparticle uptake may occur through a variety of active endocytic mechanisms, which depend on the physicochemical features of the nanoparticle and the nature of the target cells. For the successful development of nanocarriers, it is crucial to understand the molecular mechanisms involved in their interactions with the cell membrane in addition to their entry via endocytic pathways and subsequent intracellular fate. $^{2-4}$
Generally, endocytosis can be divided into two broad categories: phagocytosis (uptake of large particles) and pinocytosis (uptake of fluids, solutes, and ligands via plasma membrane receptors). Phagocytosis is characteristic of specialized professional phagocytes, whereas pinocytosis is present in virtually all cells and has multiple forms depending on the cell origin and function. ${ }^{5}$ Several different classifications for pinocytosis have been proposed, and a common approach is to order these according to the key proteins involved: clathrinmediated endocytosis (CME), caveolae-mediated endocytosis (CvME), clathrin- and caveolae-independent endocytosis, and macropinocytosis. Clathrin- and caveolae-independent pathways can be further subclassified as Arf6-, flotillin-1, Cdc42-, and RhoA-dependent endocytosis. ${ }^{5-9}$

Received: November 25, 2014

Revised: April 7, 2015

Accepted: April 16, 2015

Published: April 16, 2015 
The uptake mechanism of a drug delivery system is likely to influence its intracellular fate and capacity to mediate a biological response. The aim of the present study was to identify the endocytic mechanisms responsible for the internalization of glycol chitosan nanogels functionalized with folate in HeLa (cervical adenocarcinoma) cells that overexpress folate receptors. ${ }^{10}$ Conventionally, chemical endocytosis inhibitors have been used to analyze cellular uptake of drug delivery vectors including chitosan nanoparticles, but these inhibitors are associated with problems related to toxicity and low specificity. ${ }^{11-13}$ Selective inhibition of different endocytic pathways can also be attempted by siRNA targeting and subsequent depletion of key proteins that orchestrate individual pathways. ${ }^{14,15}$

In this work, single siRNA sequences were used to attenuate pathways regulated by clathrin heavy chain (si-CHC), caveolin1 (si-Cav-1), p21-activated kinase 1 (si-Pak-1), Flotillin-1 (siFlot-1), ${ }^{14}$ and Cdc42 (si-Cdc42). The thus-treated cells were then used to determine the mechanism of uptake and intracellular fate of the nanogels to provide valuable information regarding their capacity to deliver different types of cargos.

\section{EXPERIMENTAL SECTION}

2.1. Reagents. Glycol chitosan (GC, G7753), mercapto hexadecanoic acid (MHDA), N-hydroxysulfosuccinimide (NHS), 1-ethyl-3-[3-(dimethylamino)propyl] carbodiimide hydrochloride (EDC), O-methyl-O'-succinylpolyethyene glycol 2000 (PEG2000), O-(2-aminoethyl)-O'-(2-carboxyethyl)polyethylene glycol 3000 hydrochloride (PEG3000), folate, 3(4,5-dimethylthiazol-2-yl)-2,5-diphenyl tetrazolium bromide (MTT), and sulforhodamine B (SRB) were purchased from Sigma (St. Louis, MO, US). Folate-free RPMI 1640 medium, Opti-MEM, oligofectamine, Alexa Fluor 647 Dextran (MW = 10.000, Alexa647-dextran), Alexa Fluor 647 transferrin (Alexa647-transferrin), and Alexa Fluor 488 carboxylic acid (succinimidyl ester; Alexa488) were bought from Invitrogen (Carlsbad, CA, USA). Complete protease-inhibitor-cocktail mini tablets were from Roche Diagnostics (Mannheim, Germany). Single siRNA sequences of $21-23$ residues were acquired from Europhins MWG Operon (Ebesburg, Germany) as previously described. ${ }^{14}$

2.2. Antibodies. Antibodies recognizing clathrin heavy chain were from NeoMarker (California, US). Anti-Cav-1 was from Cell Signaling Technology (Hertfordishire, UK). AntiPak-1 was from Cell Signaling (Danvers, Massachusetts, US), and antibody specific to Flot-1 was from BD Bioscience (Oxford, UK). Anti- $\gamma$-tubulin was from Sigma (Dorset, UK). Secondary goat antimouse- and goat antirabbit-HRP antibodies were from Pierce (Loughborough, UK).

2.3. Cell Culture. HeLa cancer cells were cultured in DMEM supplemented with $10 \%$ fetal bovine serum (FBS), 100 $\mathrm{IU} / \mathrm{mL}$ penicillin, and $0.1 \mathrm{mg} / \mathrm{mL}$ streptomycin. The cells were maintained as a subconfluent monolayer in a humidified atmosphere containing $5 \% \mathrm{CO}_{2}$ at $37{ }^{\circ} \mathrm{C}$.

2.4. Self-Assembly of Nanogels. Details of GC nanogel synthesis and characterization as folic acid functionalized nanogels were described in a previous report. ${ }^{16}$ Briefly, nanogel synthesis was performed in two independent steps. Initially, folate is conjugated to PEG3000 (FA-PEG3000). In the second reaction, FA-PEG3000, PEG2000, and MHDA were grafted onto the GC polymer. The nanogel dispersions used in the different experiments were obtained after dispersing the lyophilized reaction product in distilled water under magnetic stirring at $50{ }^{\circ} \mathrm{C}$ for $48 \mathrm{~h}$ and passing the product through a pore size $0.45 \mu \mathrm{m}$ cellulose acetate syringe filter.

2.5. Preparation of the Alexa488-Labeled Nanogel. The nanogels were labeled with Alexa488 through an amide linkage. Alexa488 was dissolved in DMSO. The molar ratio of Alexa488 carboxylic groups to the nanogel free amine groups was 0.11 . The dye was added to nanogel dispersions at $1 \mathrm{mg} /$ $\mathrm{mL}$ in PBS and incubated in the dark at room temperature for $24 \mathrm{~h}$. Thereafter, the reaction mixture was extensively dialyzed (MW cutoff $=10-12 \mathrm{kDa}$ ) against distilled water to remove free Alexa488. To ensure the absence of free dye, the conjugated Alexa488-nanogel was purified by centrifugation at $3000 \mathrm{~g}$ through a $10 \mathrm{kDa} \mathrm{MW}$ cutoff filter.

2.6. Cellular Uptake of Nanogels by Flow Cytometry. HeLa cells were seeded onto 24 -well plates at $2.0 \times 10^{5}$ cells per well and left to adhere overnight. The cells were treated with nanogels at 0.2 and $0.4 \mathrm{mg} / \mathrm{mL}$ for $0,1,3,7$, and $24 \mathrm{~h}$. After each time point, the mixture, culture medium, and nanogel suspensions were removed, and the cells were washed with PBS and collected using $150 \mu \mathrm{L}$ of trypsin/EDTA (0.25\%/ $0.02 \%$ ) in PBS, applied for $2 \mathrm{~min}$ at $37{ }^{\circ} \mathrm{C}$; after addition of FBS-supplemented medium, the cell suspension was centrifuged at $300 \mathrm{~g}$ for $10 \mathrm{~min}$ and rinsed with PBS. The cellassociated fluorescence was measured by flow cytometry using a Coulter Epics XL Flow Cytometer (Beckman Coulter Inc., Miami, FL, USA).

2.7. siRNA Transfection. The transfections were performed as described by Soraj et al. ${ }^{14}$ Briefly, cells were seeded in antibiotic-free medium at a density of $1.6 \times 10^{5}$ cells per well in a $35 \mathrm{~mm}$ glass-bottomed imaging dish (MatTek, Ashland, USA) and in a 6-well plate. For 12-well plates, the density was reduced to $6.7 \times 10^{4}$ cells per well. The cells were cultured overnight to obtain the desired confluency at the beginning of the transfection $(\sim 60 \%)$. The siRNA transfection procedure was adjusted according to well diameter. Volumes used for a 12-well plate were: $0.5 \mu \mathrm{L}$ of $50 \mu \mathrm{M}$ stock siRNA diluted in $89.5 \mu \mathrm{L}$ of Opti-MEM and $2.0 \mu \mathrm{L}$ of oligofectamine in $8.0 \mu \mathrm{L}$ of Opti-MEM. The diluted solutions were then gently mixed and stored at room temperature for $30 \mathrm{~min}$. For each well, the media was removed and replaced with $400 \mu \mathrm{L}$ of Opti-MEM. The siRNA-oligofectamine complex was added dropwise to the wells and incubated at $37{ }^{\circ} \mathrm{C}$ and $5 \% \mathrm{CO}_{2}$ for $4 \mathrm{~h}$. Thereafter, $250 \mu \mathrm{L}$ of Opti-MEM containing $30 \%$ (v/v) of FBS was added to the transfection mixture, and the cells were incubated under tissue culture conditions for $48 \mathrm{~h}$.

2.8. In Vitro Viability of the Transfected Cells. 2.8.1. MTT Assay. The effect of siRNA transfection on cell metabolic activity was evaluated using the quantitative colorimetric MTT assay. The cells were seeded onto 24-well cell culture plates at a density of $3.4 \times 10^{4}$ cells per well and transfected with the different siRNAs as described above. After the transfection period $(48 \mathrm{~h})$, cell metabolic activity was measured by adding MTT (3-(4,5-dimethylthiazol-2-yl)-2,5diphenyl tetrazolium bromide). ${ }^{17}$ The MTT solution $(0.5 \mathrm{mg} /$ $\mathrm{mL}$ in PBS) was carefully removed from each well and the resulting dark blue formazan crystals were solubilized in dimethyl sulfoxide and quantified spectrophotometrically at $570 \mathrm{~nm}$. A reference absorbance at $690 \mathrm{~nm}$ was measured for the purposes of background subtraction.

2.8.2. SRB Assay. Cell proliferation of the siRNA-transfected cells was assessed using the SRB assay, which provides an estimate of total protein which in turn is related to cell 


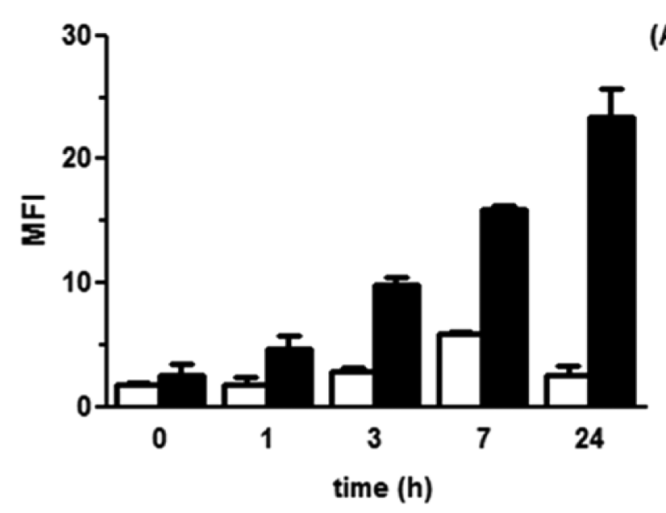

$0.2 \mathrm{mg} / \mathrm{mL}$
(A)

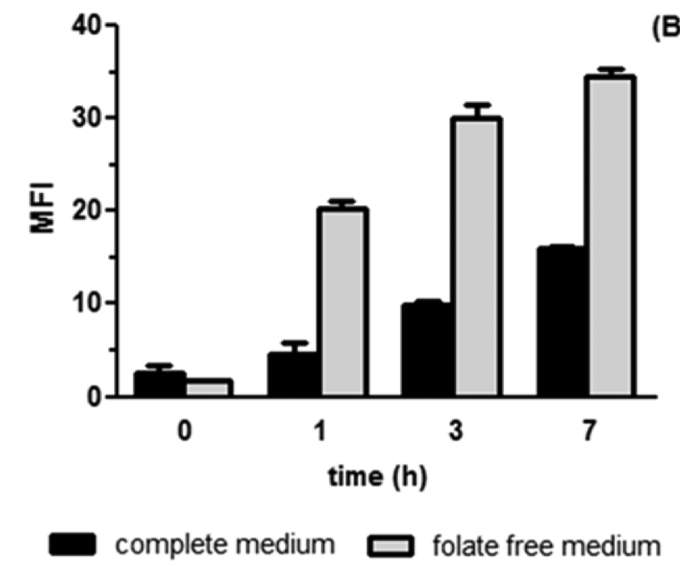

(B)

Figure 1. Effects of (A) nanogel concentration and (B) the presence of folate in the culture medium on the internalization of Alexa488-nanogel at different time points in HeLa cells. Mean fluorescence intensity (MFI) was used as a proxy for fluorescent nanogel uptake and was measured by flow cytometry at various time points up to $24 \mathrm{~h}(\mathrm{~A})$ and $7 \mathrm{~h}(\mathrm{~B})$.

number. $^{18,19}$ After transfecting cells with siRNAs for $48 \mathrm{~h}, 24$ well plates were rinsed with PBS and left to dry at $37^{\circ} \mathrm{C}$ under $5 \% \mathrm{CO}_{2}$. Ice-cold $1 \%$ acetic acid/100\% methanol solution was then used to fix the cells at $-20^{\circ} \mathrm{C}$ for $30 \mathrm{~min}$. After discarding the fixative solution, the plates were left dry at $37{ }^{\circ} \mathrm{C}$ before adding $250 \mu \mathrm{L}$ of $0.5 \%$ SRB in $1 \%$ acetic acid to each well. At $90 \mathrm{~min}$ later, the cells were washed four times with $1 \%$ acetic acid to remove the excess SRB and then left dry. A $1.0 \mathrm{~mL}$ aliquot of $10 \mathrm{mM}$ Tris solution was used to dissolve the SRB. The supernatant was used for quantification of SRB protein staining that was quantified by a spectrophotometer at $540 \mathrm{~nm}$.

2.9. Nanogel Internalization in Endocytosis-Compromised Cells. 2.9.1. Fluorescence Microscopy. 2.9.1.1. LiveCell Imaging via Confocal Microscopy. siRNA-transfected cells at $48 \mathrm{~h}$ post transfection (Section 2.7) in glass-bottomed $35 \mathrm{~mm}$ culture dishes were incubated with nanogels at $0.2 \mathrm{mg} /$ $\mathrm{mL}$ for $7 \mathrm{~h}$. The cells were then rinsed extensively with PBS and immediately imaged as live cells in phenol-red-free DMEM by confocal microscopy at $37{ }^{\circ} \mathrm{C}$ on a Leica SP5 system as previously described. ${ }^{14}$ Control and CHC-depleted cells were also incubated for $16 \mathrm{~min}$ with the CME probe Alexa647transferrin $(50 \mathrm{nM})$ to evaluate the efficiency of transfection.

2.9.1.2. Immnunolabeling. Cav-1 depletion was confirmed by immunolabeling and confocal microscopy. At the end of the transfection, the cells on glass coverslips were rinsed with PBS and fixed with $3 \%$ PFA for $15 \mathrm{~min}$ at room temperature. Then, the samples were treated with $50 \mathrm{mM}$ of $\mathrm{PBS} / \mathrm{NH}_{4}$ for $10 \mathrm{~min}$ to quench reactive species resulting from fixation. After PBS washing $(3 X)$, the cells were permeabilised with PBS $/ 0.2 \%$ Triton X-100 and washed and immersed in blocking buffer $(2 \%$ (v/v) fetal calf serum and $2 \%(\mathrm{w} / \mathrm{v})$ BSA in PBS) for $30 \mathrm{~min}$. Cells were then incubated with the primary antibody recognizing Cav-1 and subsequently with Alexa546-labeled secondary antibody. The cells were finally incubated in Hoechst 33342 for $10 \mathrm{~min}$ at room temperature to label the nucleus. Finally, the coverslips were mounted on glass sides, and the cells were visualized by confocal microscopy. Cells were imaged through the $z$ axis to generate $z$-projection images.

2.9.2. Flow Cytometry. Quantification of cell-associated fluorescence in siRNA-transfected cells following incubation with nanogel was performed by flow cytometry. For this, siRNA-transfected cells in a 12 -well plate were incubated with Alexa488-nanogel $(0.2 \mathrm{mg} / \mathrm{mL})$ for $7 \mathrm{~h}$ under tissue culture conditions. The cells were then thoroughly washed with PBS and trypsinised with trypsin/EDTA $0.25 \% / 0.02 \%$ in PBS for 2 min at $37{ }^{\circ} \mathrm{C}$; after addition of FBS-supplemented medium, the cell suspension was centrifuged at $300 \mathrm{~g}$ for $10 \mathrm{~min}$. The cell suspension was washed with PBS. Cell-associated fluorescence of the cell suspension was measured using a Coulter Epics XL Flow Cytometer (Beckman Coulter Inc., Miami, FL, USA).

2.9.3. SDS PAGE and Western Blotting. Following $48 \mathrm{~h}$ of siRNA transfection, the cells from 6-well plates were washed in ice-cold PBS and collected on ice by scraping in $100 \mu \mathrm{L}$ of lysis buffer ( $50 \mathrm{mM}$ Tris- $\mathrm{HCl}, 150 \mathrm{mM} \mathrm{NaCl}, \mathrm{pH}$ 8.0, 1\% Triton X$100)$ containing protease inhibitor cocktail. Lysates were then centrifuged at $13000 \mathrm{~g}\left(4{ }^{\circ} \mathrm{C}\right)$ for $10 \mathrm{~min}$ before adding three parts supernatant to one part $4 \times$ Laemmli buffer. The samples were heated at $95{ }^{\circ} \mathrm{C}$ for $5 \mathrm{~min}$ and centrifuged at $13000 \mathrm{~g}(4$ ${ }^{\circ} \mathrm{C}$ ) for $1 \mathrm{~min}$ prior to loading onto $10 \%$ acrylamide gels for separation by SDS-PAGE (18 $\mu \mathrm{g}$ of protein per well). Following electrophoresis, the separated proteins were transferred to PVDF membranes that were rinsed with PBS and blocked in 5\% milk in PBS/Tween 20 (0.025\%; PBST) for $1 \mathrm{~h}$. The membranes were then probed with antibodies recognizing CHC, Cav-1, Pak-1, Flot- $1, \gamma$-tubulin, and $\beta$-actin diluted in $2 \%$ milk in PBST. Following washing in PBST, species-specific secondary antibodies conjugated to horseradish peroxidase (HRP) and diluted in 2\% milk in PBST were applied to the membrane. Protein bands were detected by enhanced chemiluminescence (ECL).

2.10. Nanogel Intracellular Localization. HeLa cells were seeded at $3 \times 10^{5}$ cells in glass-bottomed dishes and left to adhere overnight. Then, $0.2 \mathrm{mg} / \mathrm{mL}$ Alexa488-nanogel and $0.025 \mathrm{mg} / \mathrm{mL}$ Alexa647-dextran were coincubated with cells for $7 \mathrm{~h}$ in folic-acid-free medium. In the case of the Alexa488nanogels, coincubation with Alexa647-transferrin (50 nM) was performed by adding labeled transferrin complex $16 \mathrm{~min}$ before the end of the nanogel incubation period $(7 \mathrm{~h})$. The cells were extensively rinsed with PBS and immediately imaged as live cells in phenol-red-free DMEM by confocal microscopy at 37 ${ }^{\circ} \mathrm{C}$.

2.11. Statistical Analysis. Results are expressed as mean \pm SD of three independent experiments, each with $n=3$. Statistical significances were determined by applying a one-way ANOVA with a Dunnett's multiple comparison test through Prism software (GraphPad software version 5.00, USA). Significance of the results is indicated according to $p$ values (*, $p<0.05$;**, $p<0.005$; and ***, $p<0.0001$ ). 


\section{RESULTS AND DISCUSSION}

We have previously shown that folate-functionalized glycol chitosan nanogels have increased uptake over the unfunctionalized nanogels. ${ }^{16}$ Herein, we investigate the uptake and internalization properties of the folate functionalized nanogels.

3.1. Cellular Uptake of Nanogels as a Function of Time, Concentration, and Presence of Folate. We examined the effects of folic acid functionalized nanogel concentration and the presence of folate in the culture medium on the internalization of Alexa488-nanogel at different time points. HeLa cells were incubated for different time periods with the fluorescent nanogels at concentrations of 0.2 and 0.4 $\mathrm{mg} / \mathrm{mL}$ in media either depleted of or containing folate. Cells were collected, and the mean fluorescence intensity (a proxy for nanogel uptake) was measured by flow cytometry.

Figure 1A shows that nanogel uptake increases with time over a $24 \mathrm{~h}$ period in cells incubated with $0.4 \mathrm{mg} / \mathrm{mL}$ nanogel. However, in cells incubated with $0.2 \mathrm{mg} / \mathrm{mL}$ nanogel, uptake increases over the first $7 \mathrm{~h}$ but then at some time during the 7$24 \mathrm{~h}$ incubation period, the mean fluorescence intensity decreases. The data indicate not only reduced uptake at this lower concentration but also a loss of the nanogel from the cells. This suggests that during longer incubation periods $(>7$ h) at this concentration the fluorescently labeled nanogel may either be recycled out of the cell or degraded. Degradation would seem more likely because recycling would potentially lead to reuptake of the nanogel, so we would expect to see stabilization in the level of internalized nanogel rather than loss.

Figure 1B demonstrates that internalization of the Alexa488nanogel is significantly increased under folate-depleted culture conditions compared with that of culture in normal media. The presence of free folate in the culture medium has previously been shown to lead to a decrease in uptake of folate-conjugated nanoparticles by cells overexpressing folate receptors. 7,20 Viewed in this context, we suggest that the folate receptors may be involved in the internalization of the Alexa488nanogel, although other nonspecific mechanisms may also contribute to the uptake. To eliminate the inhibitory and potentially confounding effects of folate in the media, folate was eliminated from subsequent uptake experiments.

3.2. Inhibition of Endocytic Pathways through siRNA Depletion of Endocytic Proteins. 3.2.1. Viability of siRNATransfected Cells. The mechanism of nanogel internalization was studied using siRNA transfection as a tool to silence endocytic proteins and inhibit distinct endocytic pathways. It is known that the level of cellular toxicity caused by transfection is dependent on the reagent used and on the nature of the cells. Cell proliferation and metabolic activity of $\mathrm{HeLa}$ cells transfected using oligofectamine were studied to ensure that viability was not compromised by the procedure. The metabolic activity of HeLa cells was not significantly affected by transfection (Figure 2A). Although statistically significant differences were detected in total protein levels, these are relatively small and unlikely to be of biological significance (Figure 2B).

3.2.2. Cellular Uptake Mechanism(s) of the Nanogels by Endocytosis-Compromised Cells. 3.2.2.1. Inhibition of Clathrin-Mediated Endocytosis. Clathrin-mediated endocytosis (CME) is the best-characterized mechanism of endocytosis and requires the Adaptor- 2 complex in association with clathrin heavy and light chain. ${ }^{7,8}$ Cargos taken up by clathrin-coated vesicles follow the classical endocytic pathway to early
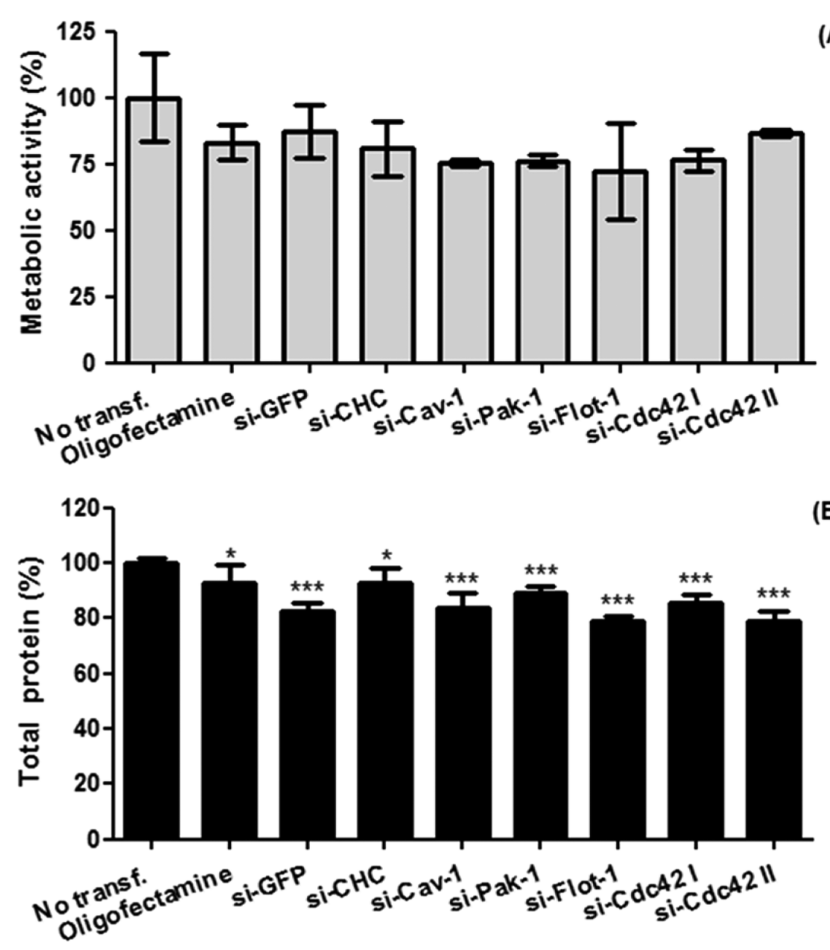

Figure 2. Effect of the siRNA transfection on metabolic activity and total protein mass of HeLa cells as assessed by the (A) MTT and (B) sulforhodamine assays. $*, p<0.05$, or $* * *, p<0.001$, represent the statistical significance of differences in viability between nontransfected cells and the siRNA-depleted samples. Results are expressed as mean $\pm \mathrm{SD}$.

endosomes where sorting occurs for delivery to other organelles such as the lysosomes or back to the cell surface through recycling endosomes. ${ }^{13}$ The uptake and recycling of transferrin occurs through this endocytic pathway; hence, this protein is commonly used as a marker to detect interference with $\mathrm{CME} .{ }^{14,21} \mathrm{CHC}$ is the major coat protein associated with this internalization pathway; ${ }^{7}$ thus in the present study, siCHC-transfected HeLa cells were incubated with Alexa647transferrin to evaluate the efficiency of CME inhibition. Transferrin uptake in clathrin-depleted cells was visibly lower compared with that of nontransfected and si-GFP transfected cells (Figure 3A), reflecting successful and population-wide transfection. CHC depletion was directly confirmed through $\mathrm{CHC}$ expression analyses in cell lysates (Figure 3B). To ascertain if the nanogel utilizes this internalization pathway, si$\mathrm{CHC}$-transfected HeLa cells and the respective controls were incubated with nanogels and then analyzed via confocal microscopy (Supporting Information Figure 1) and flow cytometry (Figure 3C), revealing no differences in nanogel uptake between control cells and those deficient in CME, suggesting that this pathway is not involved in nanogel uptake.

3.2.2.2. Inhibition of Caveolae-Mediated Endocytosis. Caveolae-mediated endocytosis (CvME) starts with the formation of caveolae on the plasma membrane of cells, often in lipid rafts. Caveolae are noted as being enriched in the protein Cav-1 and have a diameter between 50 and $100 \mathrm{~nm}$. A dynamin-dependent scission of the caveolae from the membrane then results in the formation of the endosome-like caveosome. ${ }^{8}$ Initially, the caveosome interior was thought not to acidify to any great extent with delivery of cargo to the Golgi and/or endoplasmic reticulum, thus avoiding lysosomal 

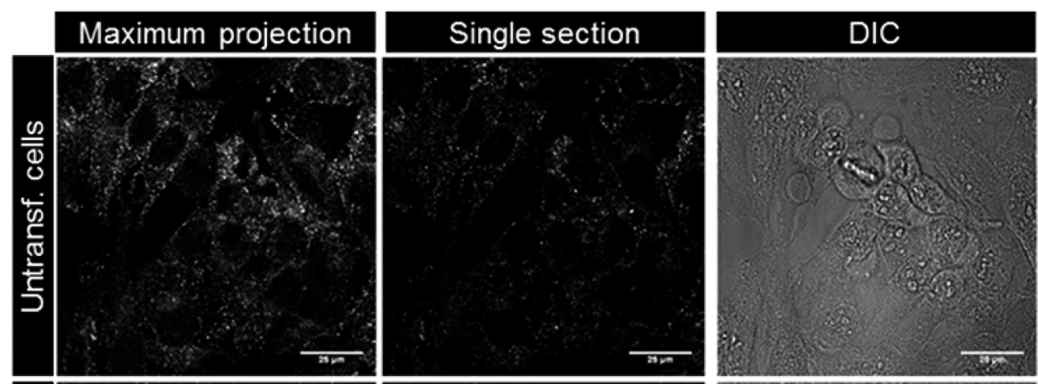

(A)

(B)
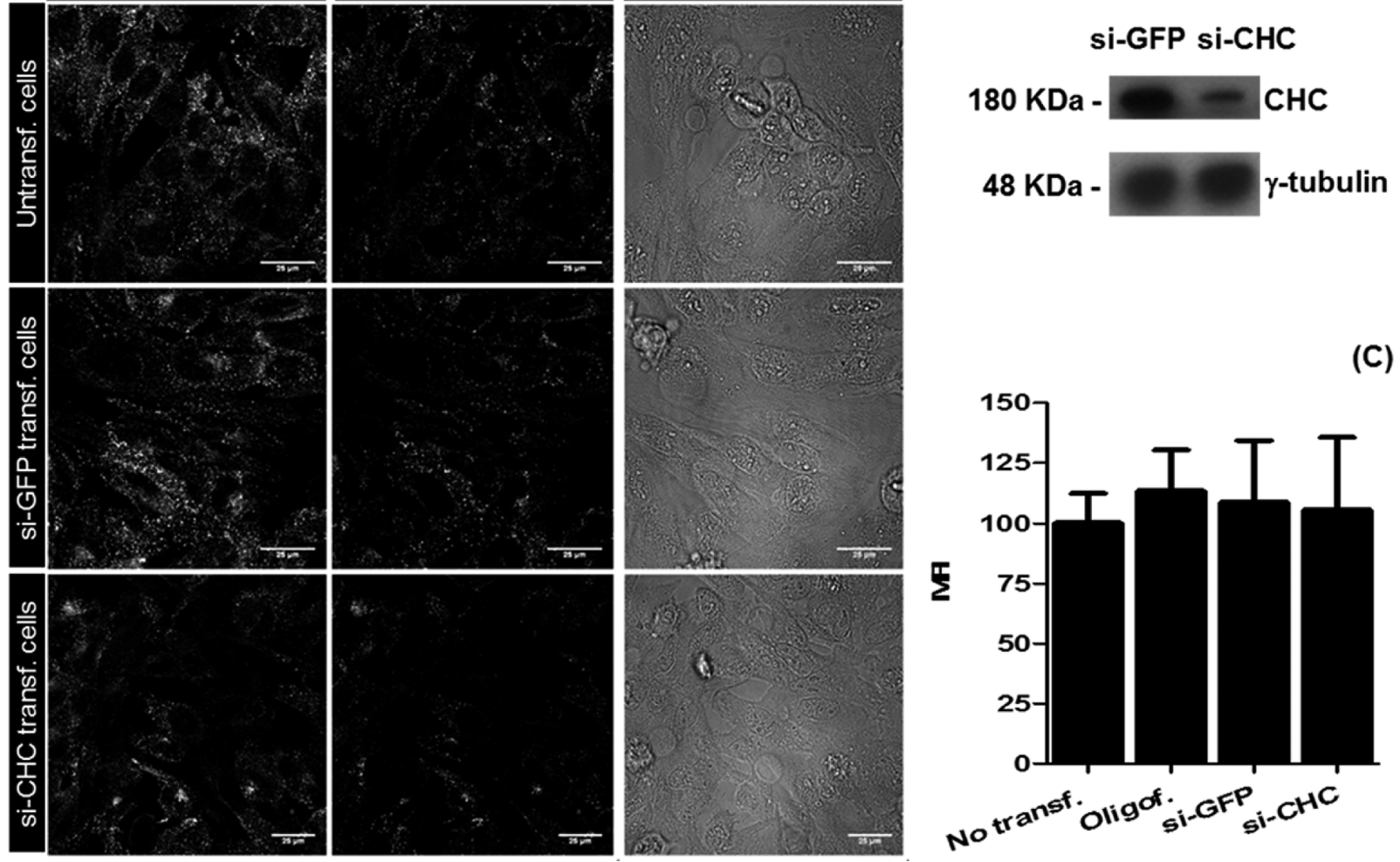

Figure 3. CME inhibition in HeLa cells. (A) Confocal visualization of HeLa cells transfected with si-CHC or si-GFP (control) for $48 \mathrm{~h}$ prior to incubation with Alexa647-transferrin for $16 \mathrm{~min}$. For each condition (rows), the images correspond to (left to right, respectively) maximum projection, middle $z$ section through the cells, and differential interference contrast (DIC) images. Scale bars $=25 \mu \mathrm{m}$. (B) CHC expression after transfecting HeLa cells with si-CHC for $48 \mathrm{~h}$. (C) Effect of the CME silencing on cellular uptake of nanogel, expressed as mean fluorescence intensity (MFI). Error bars represent SD.

degradation. ${ }^{8}$ However, Chiu et al. ${ }^{22}$ revealed that N-palmitoyl chitosan nanoparticles enter cells via caveolae and were transiently localized in caveosomes before trafficking to the canonical endosomal pathway en route to lysosomes. Agents, such as viruses, that are taken up by caveolae are also thought to end up in endosomes that can be directed to lysosomes. Evidence from electron microscopy studies suggests that caveosomes represent a special type of caveolar endosome that always associates with the plasma membrane. ${ }^{23}$ The theory behind the existence of caveosomes mostly results from studies that involve caveolin overexpression or caveolin mutants and as such may or may not be a naturally occurring phenomenon. ${ }^{23}$

CvME inhibition was achieved through depletion of Cav-1 using transfection with si-Cav-1. Because Cav-1 is a critical component of caveolae formation at the plasma membrane, ${ }^{8}$ its depletion results in the impairment of CvME. The efficiency of siRNA transfection was visualized by immunofluorescence microscopy of Cav-1 because a reliable endocytic marker equivalent to transferrin for $\mathrm{CME}$ for assessing uptake via caveolae has not yet been identified. As shown in Figure 4A, a decrease in caveolin vesicle labeling was clearly observed in siCav-1 transfected cells. Significant Cav-1 depletion was also confirmed through Western blot analysis of cell lysates (Figure 4B).

There is data to suggest that folate internalization through the folate receptor occurs via CvME. ${ }^{3}$ For example, folatetargeted poly(ethylene glycol) (PEG)-coated nanoparticles were thought to internalize via caveolae-assisted endocytosis, and the nanoparticles were subsequently visualized in punctate structures. ${ }^{24}$ Conversely, internalization and trafficking of folate receptors was found not to be exclusively caveolae-dependent: specifically, folate-protein conjugates, after binding to folate receptors on the surface of cancer cells, were internalized irrespective of size via uncoated pits or caveolae. However at later times $(6 \mathrm{~h})$, some conjugates were found in lysosomes, showing that the caveolae-mediated endocytic pathway converged with a pathway utilized by clathrin-coated pits. ${ }^{25}$ In agreement with this, as shown by confocal microscopy in Supporting Information Figure 2 and quantified by flow cytometry in Figure 4C, nanogel uptake was reduced, but not significantly $(p \geq 0.05)$, in Cav-1-depleted cells. However, it should be noted that when a particular route is inhibited, others may become activated as a compensatory mechanism. ${ }^{22}$ For example, Cav-1 depletion has been shown to result in increased levels of activated $\mathrm{Cdc} 42$ at the plasma membrane; ${ }^{5}$ this protein is thought to regulate the actin cytoskeleton and folate internalization through the glycosylphosphatidylinositol (GPI)-anchored folate receptor. ${ }^{26}$

The hydrophobicity of the cargo may be a determining factor for its internalization via CvME. The uptake of $\mathrm{N}$-palmitoyl chitosan nanoparticles was associated with lipid-raft-mediated endocytic routes, and substitution of chitosan with a higherlevel palmitoyl groups $(>10 \%)$ increased the fraction of nanogel uptake via CvME. ${ }^{22}$

Also noteworthy is the impact of nanogel size distribution on CvME and indeed on other pathways. The nanogels in this study have mean diameters of around $200 \mathrm{~nm}$, and size may play a critical role in their mechanism of uptake. To our 


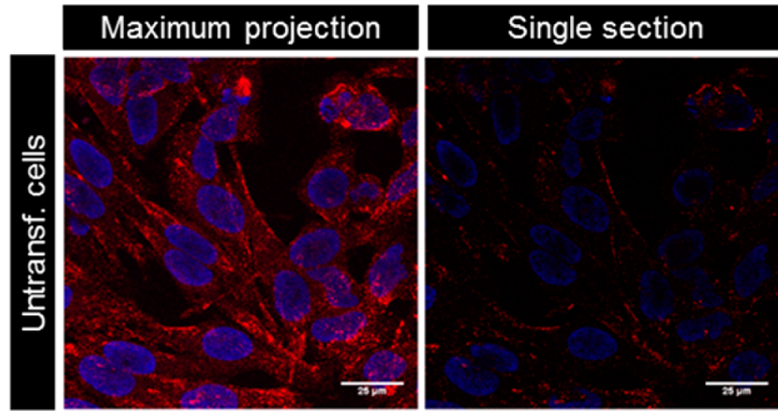

(A)

(B)
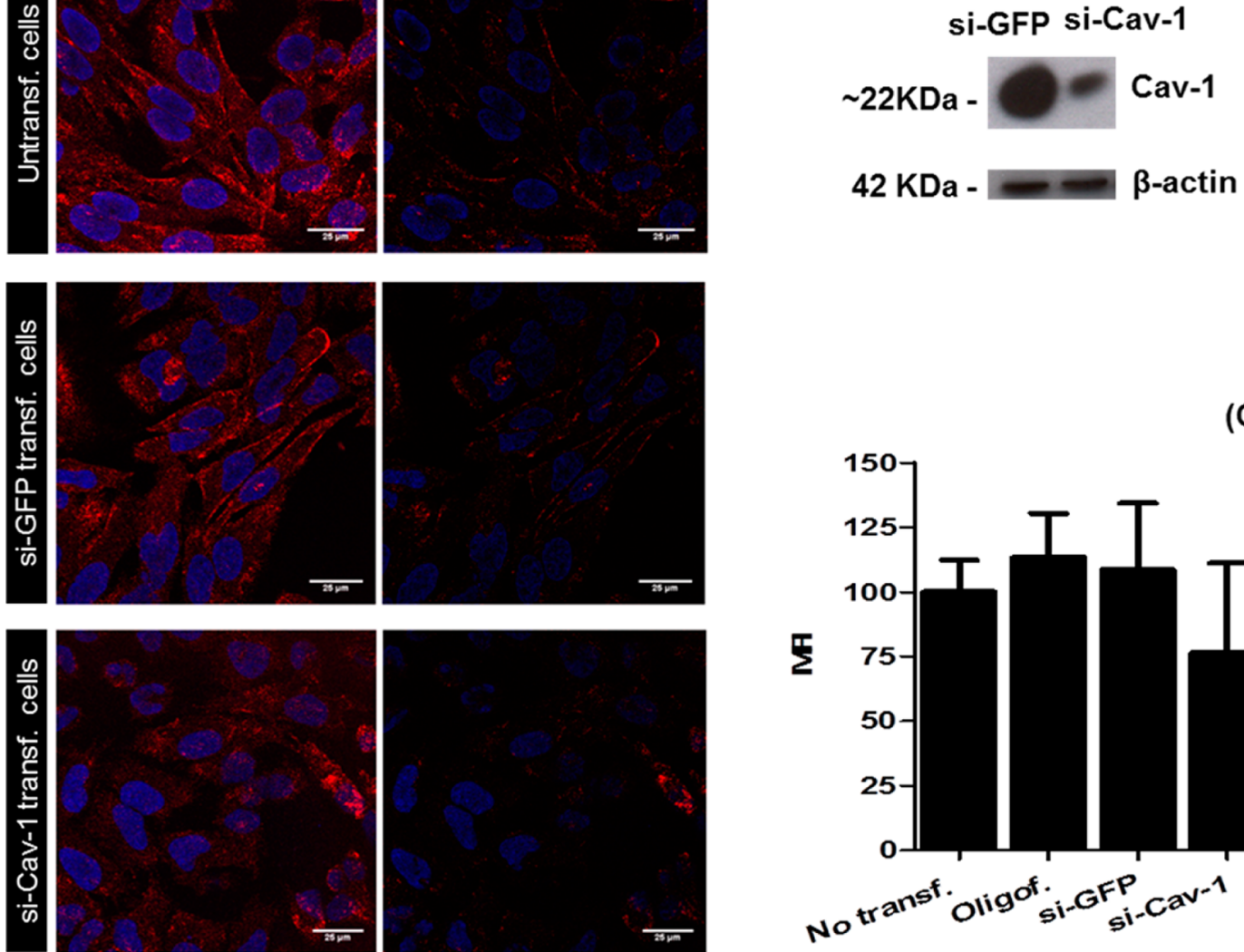

(C)

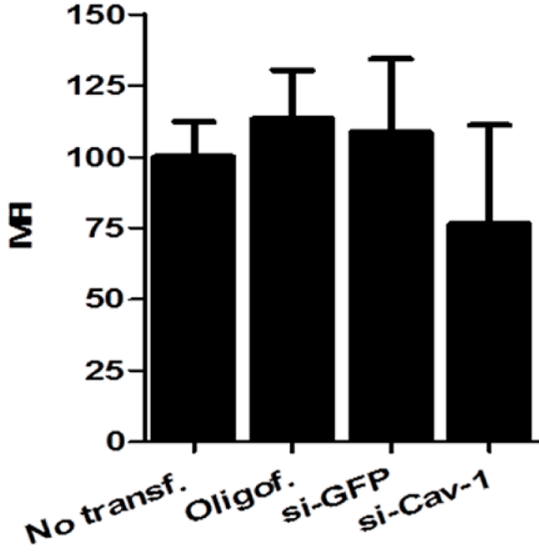

Figure 4. CvME inhibition in HeLa cells. (A) Control and si-Cav-1- or GFP-treated cells were fixed after 48 h, labeled with antibodies against Cav-1 and with Alexa546-conjugated secondary antibody, and then analyzed by confocal fluorescence microscopy. Maximum projection (left) and a single $z$ section (right) is shown for each condition; nuclei are labeled with Hoechst 33342. Scale bars $=25 \mu \mathrm{m}$. (B) Cav-1 expression after transfecting HeLa cells with si-Cav-1 for $48 \mathrm{~h}$. (C) Effect of the CvME inhibition on cellular uptake of nanogels expressed as mean fluorescence intensity (MFI). Error bars represent SD.

knowledge, no studies have unambiguously demonstrated that caveolae can accommodate particles larger than $100 \mathrm{~nm}$, and this has previously been noted. ${ }^{27}$ However, CvME was thought to play a major role in the cellular uptake of chitosan/DNA/ $\operatorname{poly}(\gamma$-glutamic acid) complexes with an average size of approximately $150 \mathrm{~nm} .{ }^{28}$ It should be noted that most studies suggesting that caveolae are involved in the uptake of drug delivery vectors rely on the use of chemical endocytosis inhibitors that often influence more than one pathway. ${ }^{12}$

3.2.2.3. Inhibition of Macropinocytosis. Macropinocytosis is a poorly characterized pathway that internalizes extracellular material in the fluid phase. This process involves, especially after growth factor activation, membrane ruffling and formation of relatively large vesicles named macropinosomes. These are heterogeneous in size, generally considered larger than $0.2 \mu \mathrm{m}$, and have no clearly identified and unique coat structures. ${ }^{29,30} \mathrm{It}$ is known that p21-activated kinase (Pak-1) is associated with growth-factor-induced macropinosomes ${ }^{14}$ and can be activated by the small GTPases Cdc42 and Rac1. ${ }^{31}$ Whether the fate of macropinosomes inside the cells involves fusion with lysosomes or recycling to the plasma membrane or a mixture of both is unclear and likely to be dependent on cell type. ${ }^{8}$

The addition of cationic molecules and particles to cells will inevitably result in interaction with the negative surface, mediated by surface sugars. ${ }^{2,3}$ This may or may not lead to internalization via membrane turnover or activation of macropinocytosis through ruffling. Membrane ruffling has been extensively studied in the field of cationic cell-penetrating peptides. ${ }^{14,30}$ In the present study, we found that the cellular uptake of the positively charged nanogel $(+25 \mathrm{mV})^{16}$ was significantly affected in Pak-1-depleted cells (Figure 5A,B).

Studies using chemical inhibitors in HeLa cells have suggested that macropinocytosis plays a crucial role in the

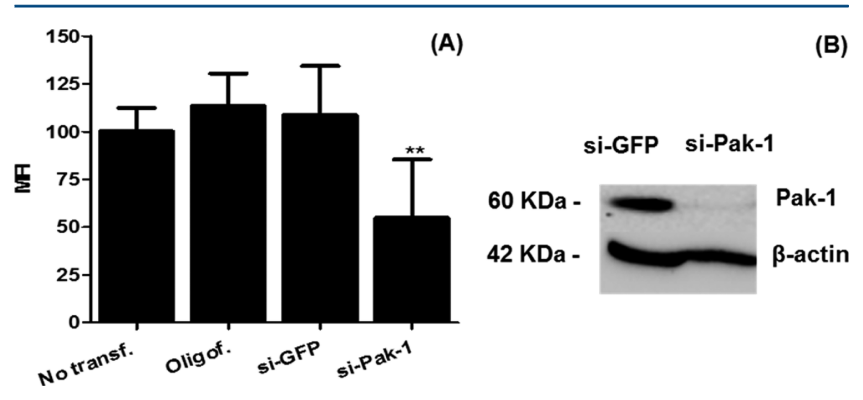

Figure 5. Pak-1 depletion of HeLa cells. (A) Inhibition of cellular uptake of nanogel, expressed as mean fluorescence intensity (MFI).** represents statistical significance of $p<0.01$ for differences in nanogel uptake between untransfected cells and si-Pak-1-transfected cells; error bars represent SD. (B) Pak-1 expression after transfecting HeLa cells for $48 \mathrm{~h}$ with control or si-Pak-1. 
internalization of hydrophobically modified glycol chitosan nanoparticles (mean size $=350 \mathrm{~nm}$ and positive surface charge $=+22 \mathrm{mV}) .{ }^{4}$ However, other uptake pathways were also thought to be involved in uptake of these nanoparticles.

3.2.2.4. Inhibition of Clathrin- and Caveolin-Independent Endocytosis: Cdc42- and Flotillin-Dependent Endocytosis. Nanoparticles and polymers modified with folate have been shown to bind to folate receptors that are often overexpressed in tumor cells. ${ }^{6,32}$ These multiple GPI-anchored proteins (GPIAps) are slowly internalized by cells and can be detected in a population of early endosomal organelles referred to as GPIenriched early endosomal compartments, or GEECs. The uptake process is not blocked by perturbations of CME. ${ }^{26,31}$ Cdc42-dependent endocytosis has been reported to be involved in the uptake of GPI-Aps, ${ }^{23}$ and because of the actin mediated effects of $\mathrm{Cdc} 42$, it is also a well characterized regulator of macropinocytosis. ${ }^{29}$ Raft-associated proteins flotillin-1 and flotillin-2 are also thought to play a role in cellular uptake and trafficking mechanisms of nanoparticles. Flotillin-1 has been described as regulator of specific clathrin- and caveolaeindependent uptake mechanisms. ${ }^{15,23}$ Furthermore, flotillins appear to play a general function in late or lysosomal degradation or storage processes. ${ }^{33}$

To investigate whether the nanogel internalization is affected by flotillin-1 and/or Cdc42-dependent pathways, expression of these proteins in HeLa cells was siRNA depleted prior to performing uptake assays. The results shown in Figure 6A

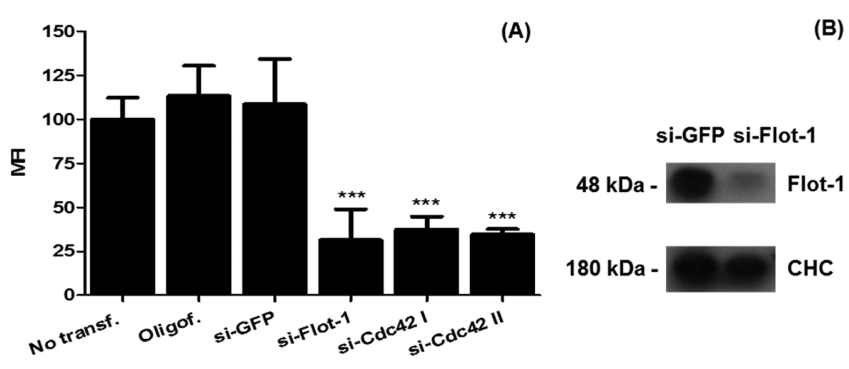

Figure 6. (A) Flow cytometry analysis of the HeLa cells depleted of flot-1 or Cdc42 and incubated with Alexa488-nanogel for $7 \mathrm{~h}$. Error bars represent $\mathrm{SD} ; * * *$ indicates a statistical significance of $p<0.001$ for the differences in nanogel uptake between untransfected cells and the siRNA-transfected cells. Error bars represent SD. (B) Flotillin-1 expression after transfecting HeLa cells for $48 \mathrm{~h}$ with siRNA targeting Flot-1. CHC expression was measured as a loading control.

demonstrate that cellular uptake is significantly affected in these endocytosis compromised cells: nanogel uptake was inhibited by approximately 70 and $65 \%$ in cells depleted of flotillin-1 (Figure 6B) or Cdc42, respectively. We are currently unable to label $\mathrm{Cdc} 42$ on membranes to investigate the extent of protein depletion, but importantly, two different sequences targeting Cdc42 were shown to have almost identical effects on nanogel uptake.

The complete siRNA data presented in this study are summarized in Figure 7, highlighting that the nanogel internalization appears to be dependent on multiple endocytic pathways. This has been previously reported for chitosan and chitosan-derived nanoparticles using chemical inhibitors of endocytosis. $^{13,34}$ It remains to be determined whether the nanogel is actually entering via a flotillin- or Cdc42-mediated pathway or even macropinocytosis, but it raises interesting questions regarding the effect of depletion of these proteins on the overall organization of the plasma membrane. The effects of

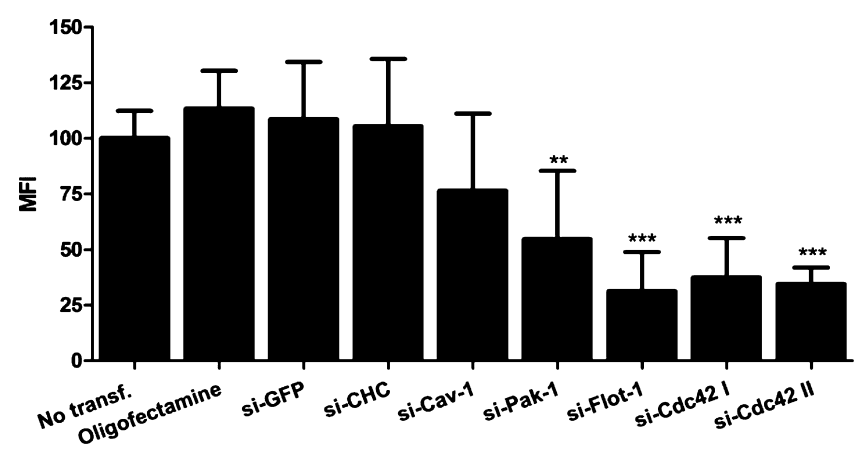

Figure 7. Mean fluorescence intensity (MFI) of the nanogel internalized by HeLa cells transfected with si-CHC, si-Cav-1, si-Pak1, si-Flot-1, and si-Cdc42, as measured by flow cytometry. Untreated cells and cells incubated with oligofectamine alone or transfected with oligofectamine/si-GFP were tested as negative controls. At $48 \mathrm{~h}$ posttransfection, the cells were incubated with nanogel, trypsinised, and analyzed by flow cytometry. $* *, p<0.01$, or $* * *, p<0.001$, represent the statistical significance of differences in nanogel between untransfected cells and the remaining samples. Error bars represent SD.

depletion of Cdc42 and Pak-1 also highlight the importance of the actin cytoskeleton and most probably macropinocytosis in the uptake of the nanogels. We suggest that nanogels enter the cell through their activation of the cell membrane, promoting their own uptake via actin reorganization and membrane ruffling. Further experiments are required to test this hypothesis more thoroughly.

Using siRNA to overcome the lack of specificity associated with traditional chemical inhibitors, we are able to more accurately target endocytic pathways utilized by the nanogel. Different pathways can be utilized to direct cargo to various cellular destinations including the lysosomes, Golgi, or exocytic structures for recycling. Overall, determining the uptake mechanism utilized by a nanoparticle can significantly aid in the understanding of the nanoparticle's ability to perform its function and provide information for rational design of improved delivery systems.

3.3. Nanogel Intracellular Localization. Once the key mechanisms of nanogel internalization had been identified, we aimed to investigate their intracellular fate by confocal laser scanning microscopy. Determining the subcellular localization of these vectors at different time points can give important information regarding their suitability for delivering therapeutic cargo into cells and specific subcellular compartments. For this, we made use of well-characterized endocytic probes whose mechanism of uptake is understood, as is their eventual cellular fate. The intracellular localization of the Alexa488-nanogel was compared with transferrin and dextran, both labeled with Alexa647. By using specific experimental conditions, these probes can be used to identify early, recycling, and late endosomes as well as terminal lysosomes. ${ }^{21,35}$

When internalized, iron-loaded holo-transferrin is trafficked via clathrin-coated vesicles to early endosomes and then further directed to recycling endosomes. Finally, it is returned to the cell surface as apo-transferrin for another constitutive cycle of iron loading. ${ }^{21}$ To determine if the nanogels enter the same early/recycling endosomal compartment as transferrin, we coincubated the two compounds for the final $16 \mathrm{~min}$ of the $7 \mathrm{~h}$ nanogel uptake period prior to performing live-cell confocal microscopy. This short period allows the transferrin to traffic to, and so label, early and recycling endosomes. The images and 

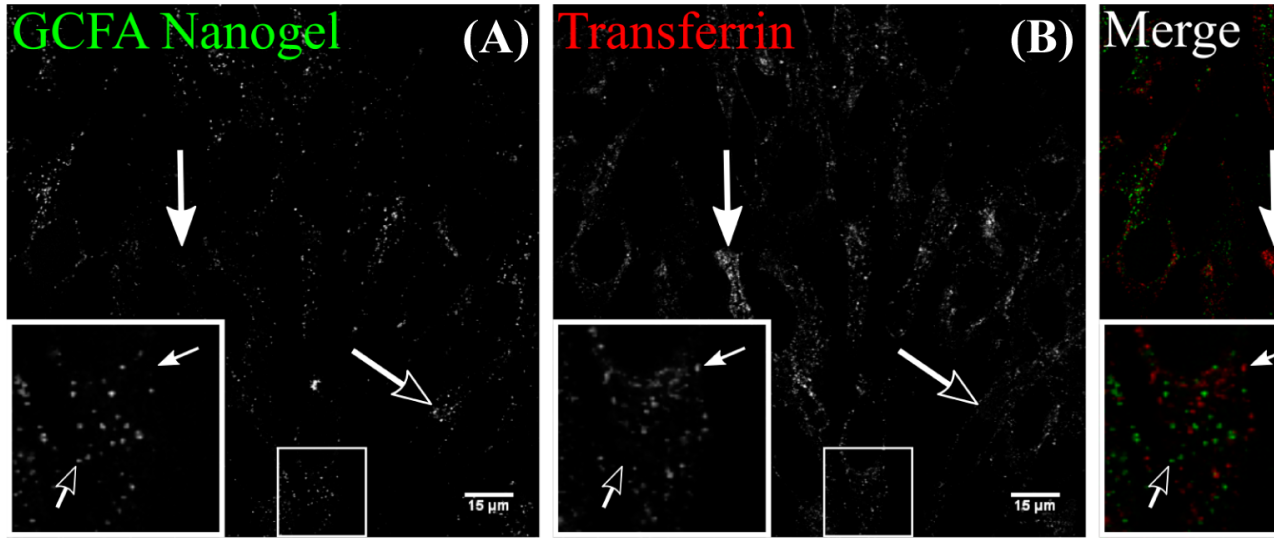

(C)
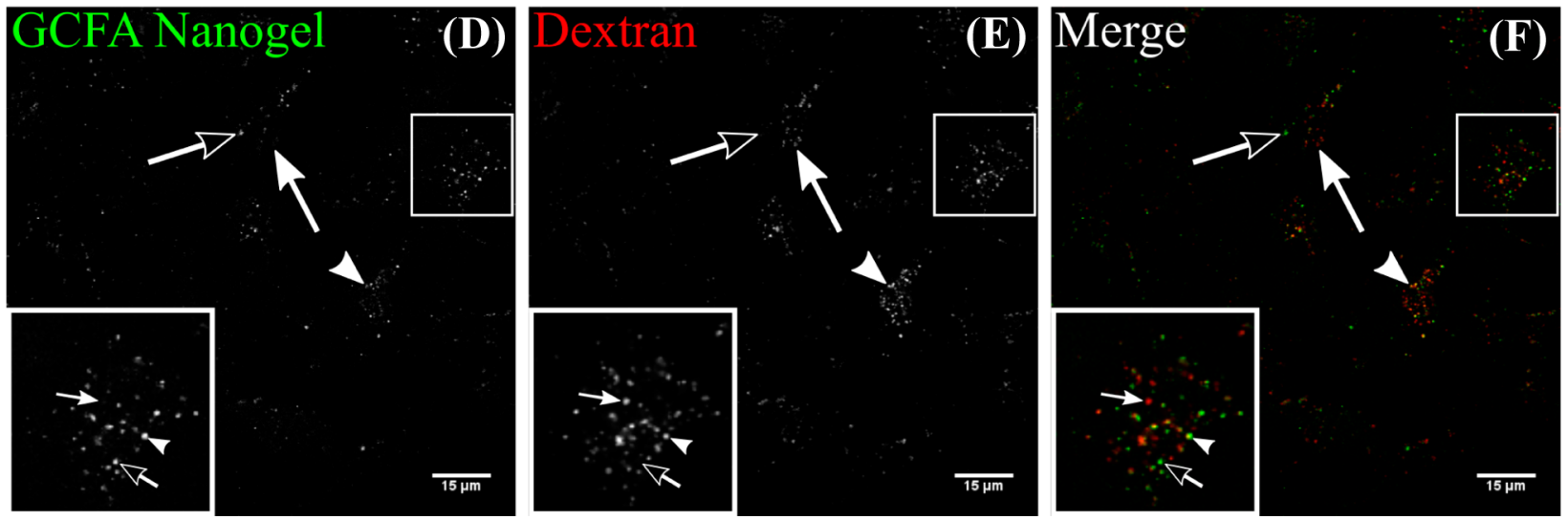

Figure 8. Subcellular distribution of nanogel in HeLa cells after $7 \mathrm{~h}$ of incubation with (A-C) Alexa647-transferrin (16 min prior to analysis) or (D-F) Alexa647-dextran (7 h coincubation). The images correspond to single-channel capture of nanogels (A and D, green in merge), transferrin (B, red in merge), and dextran (E, red in merge) and respective merged images ( $\mathrm{C}$ and $\mathrm{F}$ ). Unfilled arrows show single nanogel structures, filled arrows show single transferrin or dextran structures, and arrowheads show colocalization between nanogel and probe. Scale bars $=15 \mu \mathrm{m}$.

colocalization data shown in Figure 8A-C demonstrate that there is very little colocalization between transferrin and the nanogel after they have entered the cell. This is expected on the basis of data in Figure 3 and also the possibility that most of the nanogels have trafficked to other endocytic compartments that would be devoid of transferrin labeling, i.e., late endosomes and lysosomes.

To test whether the nanogel was in fact being trafficked via endolysosomal structures, nanogel/dextran coincubation experiments were performed. These experiments revealed considerable colocalization $(\sim 50 \%)$ between nanogel and dextran, as illustrated by colocalization of the two probes in distinct intracellular yellow dots Figure 8D-F.

$\mathrm{Cdc} 42$ has also been shown to regulate fluid-phase endocytosis, and dextran enters cells via this mechanism. ${ }^{36}$ Thus, we infer from the data in Figure $8 \mathrm{~F}$ that the nanogel colocalized with the dextran represents the fraction of nanogel that enters the cell in a Cdc42-dependent manner. This conclusion is strengthened by our findings in Figure 6 that $\mathrm{Cdc} 42$ is required for $\sim 70 \%$ of nanogel uptake. The absence of complete colocalization may be attributed to the fraction that enters the cell via lipid rafts and is thus regulated by the expression of flot-1. Previous studies by other researchers have demonstrated a high extent of colocalization between acidic organelles and hydrophobically modified glycol chitosan nanoparticles in HeLa H2B-GFP cells labeled with LysoTracker Red probe. ${ }^{4}$ Other research using human renal proximal tubular KHC cells has shown that $50 \%$ of positively charged chitosanbased nanoparticles could escape from lysosomes and reach the cytosol after $6 \mathrm{~h}$ of incubation. ${ }^{37}$ Lower levels of colocalization ( 20\%) of hydrophobically modified glycol chitosan nanoparticles/lysosomal vesicles labeled with LysoTracker in HeLa cells has also been noted. ${ }^{34}$

The data from this study suggest that a fraction of nanogel may ultimately be delivered to lysosomes. This is not unexpected because pathways such as CME, caveolae, and macropinocytosis have been shown to deliver some ligands and their receptors to lysosomes. However, because molecules such as transferrin can enter cells via CME but are recycled rather than degraded, it remains to be determined what, if any, fraction of the nanogels recycles. The possibility exists that recycling could be mediated by late endocytic structures via a pathway that may be distinct from that used by transferrin. ${ }^{38}$

Although undesirable in many cases for therapeutic cargo that would be inactivated in lysosomes, e.g., siRNA or DNA, specific lysosome targeting may be beneficial for drug delivery strategies to replace deficient lysosome enzymes to treat conditions such as lysosomal storage diseases. ${ }^{39}$ Beyond this, nanomedicines targeting the endolysosomal pathway also have potential for improving drug delivery to address other major disease burdens including Alzheimer's disease and cancer. ${ }^{3,37}$

\section{CONCLUSIONS}

Inhibition of endocytic pathways via siRNA depletion of specific endocytic proteins has provided new insights into the way that nanogel enters cells with respect to the requirements for specific proteins and the types of pathways that they orchestrate. 
The involvement of Cdc42 and Pak-1 strongly suggests that actin reorganization is also required for uptake, but whether this is constitutive or active macropinocytosis remains to be determined. Colocalization studies with endocytic probes showed that some nanogel is delivered to endolysosomal compartments, but a significant fraction was also in organelles that await further characterization.

\section{ASSOCIATED CONTENT}

\section{S Supporting Information}

Live cell confocal microscopy observation of Alexa488nanogels incubated with si-CHC- and si-Cav-1-transfected cells. This material is available free of charge via the Internet at http://pubs.acs.org.

\section{AUTHOR INFORMATION}

\section{Corresponding Author}

*E-mail: fmgama@deb.uminho.pt.

\section{Notes}

The authors declare no competing financial interest.

\section{ACKNOWLEDGMENTS}

P.P. and F.M.G. thank the project BioHealth - Biotechnology and Bioengineering approaches to improve health quality, Ref. NORTE-07-0124-FEDER-000027, cofunded by the Programa Operacional Regional do Norte (ON.2 - O Novo Norte), QREN, FEDER. P.P. was funded through an FCT Ph.D. grant (SFRH/BD/64977/2009). Funding is also acknowledged from a Cancer Research UK studentship (C36040/A11652) to A.T.J. and J.M.W. and an EPSRC Grant (EP/J021334/1 to A.T.J. and E.S.).

\section{REFERENCES}

(1) Gao, H.; Yang, Z.; Zhang, S.; Cao, S.; Shen, S.; Pang, Z.; Jiang, X. Ligand modified nanoparticles increases cell uptake, alters endocytosis and elevates glioma distribution and internalization. Sci. Rep. 2013, 3, 2534.

(2) Harush-Frenkel, O.; Debotton, N.; Benita, S.; Altschuler, Y. Targeting of nanoparticles to the clathrin-mediated endocytic pathway. Biochem. Biophys. Res. Commun. 2007, 353, 26-32.

(3) Bareford, L. M.; Swaan, P. W. Endocytic mechanisms for targeted drug delivery. Adv. Drug Delivery Rev. 2007, 59, 748-58.

(4) Park, S.; Lee, S. J.; Chung, H.; Her, S.; Choi, Y.; Kim, K.; Choi, K.; Kwon, I. C. Cellular uptake pathway and drug release characteristics of drug-encapsulated glycol chitosan nanoparticles in live cells. Microsc. Res. Tech. 2010, 73, 857-65.

(5) Doherty, G. J.; McMahon, H. T. Mechanisms of endocytosis. Annu. Rev. Biochem. 2009, 78, 857-902.

(6) Sahay, G.; Alakhova, D. Y.; Kabanov, A. V. Endocytosis of nanomedicines. J. Controlled Release 2010, 145, 182-95.

(7) Xu, S.; Olenyuk, B. Z.; Okamoto, C. T.; Hamm-Alvarez, S. F. Targeting receptor-mediated endocytotic pathways with nanoparticles: rationale and advances. Adv. Drug Delivery Rev. 2013, 65, 121-38.

(8) Xiang, S.; Tong, H.; Shi, Q.; Fernandes, J. C.; Jin, T.; Dai, K.; Zhang, X. Uptake mechanisms of non-viral gene delivery. J. Controlled Release 2012, 158, 371-8.

(9) Ilina, P.; Hyvonen, Z.; Saura, M.; Sandvig, K.; Yliperttula, M.; Ruponen, M. Genetic blockage of endocytic pathways reveals differences in the intracellular processing of non-viral gene delivery systems. J. Controlled Release 2012, 163, 385-95.

(10) Wang, X.; Yao, S.; Ahn, H.-Y.; Zhang, Y.; Bondar, M. V.; Torres, J. A.; Belfield, K. D. Folate receptor targeting silica nanoparticle probe for two-photon fluorescence bioimaging. Biomed. Opt. Express 2010, 1, $453-462$.
(11) Vercauteren, D.; Vandenbroucke, R. E.; Jones, A. T.; Rejman, J.; Demeester, J.; De Smedt, S. C.; Sanders, N. N.; Braeckmans, K. The use of inhibitors to study endocytic pathways of gene carriers: optimization and pitfalls. Mol. Therapy 2010, 18, 561-9.

(12) Ivanov, A. I. Pharmacological inhibition of endocytic pathways: is it specific enough to be useful? Methods Mol. Biol. 2008, 440, 15-33.

(13) Garaiova, Z.; Strand, S. P.; Reitan, N. K.; Lelu, S.; Storset, S. O.; Berg, K.; Malmo, J.; Folasire, O.; Bjorkoy, A.; Davies Cde, L. Cellular uptake of DNA-chitosan nanoparticles: the role of clathrin- and caveolae-mediated pathways. Int. J. Biol. Macromol. 2012, 51, 1043-51.

(14) Al Soraj, M.; He, L.; Peynshaert, K.; Cousaert, J.; Vercauteren, D.; Braeckmans, K.; De Smedt, S. C.; Jones, A. T. siRNA and pharmacological inhibition of endocytic pathways to characterize the differential role of macropinocytosis and the actin cytoskeleton on cellular uptake of dextran and cationic cell penetrating peptides octaarginine (R8) and HIV-Tat. J. Controlled Release 2012, 161, 13241.

(15) Vercauteren, D.; Piest, M.; van der Aa, L. J.; Al Soraj, M.; Jones, A. T.; Engbersen, J. F.; De Smedt, S. C.; Braeckmans, K. Flotillindependent endocytosis and a phagocytosis-like mechanism for cellular internalization of disulfide-based poly(amido amine)/DNA polyplexes. Biomaterials 2011, 32, 3072-84.

(16) Pereira, P.; Morgado, D.; Crepet, A.; David, L.; Gama, F. M. Glycol chitosan-based nanogel as a potential targetable carrier for siRNA. Macromol. Biosci. 2013, 13, 1369-78.

(17) Mosmann, T. Rapid colorimetric assay for cellular growth and survival: Application to proliferation and cytotoxicity assays. $J$. Immunol. Methods 1983, 65, 55-63.

(18) Papazisis, K. T.; Geromichalos, G. D.; Dimitriadis, K. A.; Kortsaris, A. H. Optimization of the sulforhodamine B colorimetric assay. J. Immunol. Methods 1997, 208, 151-8.

(19) Skehan, P.; Storeng, R.; Scudiero, D.; Monks, A.; McMahon, J.; Vistica, D.; Warren, J. T.; Bokesch, H.; Kenney, S.; Boyd, M. R. New colorimetric cytotoxicity assay for anticancer-drug screening. J. Natl. Cancer Inst. 1990, 82, 1107-12.

(20) Liu, L.; Zheng, M.; Renette, T.; Kissel, T. Modular Synthesis of Folate Conjugated Ternary Copolymers: Polyethylenimine-graftPolycaprolactone-block-Poly(ethylene glycol)-Folate for Targeted Gene Delivery. Bioconjugate Chem. 2012, 23, 1211-1220.

(21) Mayle, K. M.; Le, A. M.; Kamei, D. T. The intracellular trafficking pathway of transferrin. Biochim. Biophys. Acta 2012, 1820, 264-81.

(22) Chiu, Y. L.; Ho, Y. C.; Chen, Y. M.; Peng, S. F.; Ke, C. J.; Chen, K. J.; Mi, F. L.; Sung, H. W. The characteristics, cellular uptake and intracellular trafficking of nanoparticles made of hydrophobicallymodified chitosan. J. Controlled Release 2010, 146, 152-9.

(23) Sandvig, K.; Pust, S.; Skotland, T.; van Deurs, B. Clathrinindependent endocytosis: mechanisms and function. Curr. Opin. Cell Biol. 2011, 23, 413-420.

(24) Dauty, E.; Remy, J. S.; Zuber, G.; Behr, J. P. Intracellular delivery of nanometric DNA particles via the folate receptor. Bioconjugate Chem. 2002, 13, 831-9.

(25) Turek, J. J.; Leamon, C. P.; Low, P. S. Endocytosis of folateprotein conjugates: ultrastructural localization in KB cells. J. Cell Sci. 1993, 106, 423-30.

(26) Sabharanjak, S.; Sharma, P.; Parton, R. G.; Mayor, S. GPIanchored proteins are delivered to recycling endosomes via a distinct cdc42-regulated, clathrin-independent pinocytic pathway. Dev. Cell 2002, 2, 411-23.

(27) Iversen, T.-G.; Skotland, T.; Sandvig, K. Endocytosis and intracellular transport of nanoparticles: Present knowledge and need for future studies. Nano Today 2011, 6, 176-185.

(28) Peng, S. F.; Tseng, M. T.; Ho, Y. C.; Wei, M. C.; Liao, Z. X.; Sung, H. W. Mechanisms of cellular uptake and intracellular trafficking with chitosan/DNA/poly(gamma-glutamic acid) complexes as a gene delivery vector. Biomaterials 2011, 32, 239-48.

(29) Kerr, M. C.; Teasdale, R. D. Defining macropinocytosis. Traffic (Oxford, U.K.) 2009, 10, 364-71. 
(30) Jones, A. T. Macropinocytosis: searching for an endocytic identity and role in the uptake of cell penetrating peptides. J. Cell. Mol. Med. 2007, 11, 670-84.

(31) Hansen, C. G.; Nichols, B. J. Molecular mechanisms of clathrinindependent endocytosis. J. Cell Sci. 2009, 122, 1713-21.

(32) Lu, Y.; Low, P. S. Folate-mediated delivery of macromolecular anticancer therapeutic agents. Adv. Drug Delivery Rev. 2002, 54, 67593.

(33) Kasper, J.; Hermanns, M. I.; Bantz, C.; Utech, S.; Koshkina, O.; Maskos, M.; Brochhausen, C.; Pohl, C.; Fuchs, S.; Unger, R. E.; Kirkpatrick, C. J. Flotillin-involved uptake of silica nanoparticles and responses of an alveolar-capillary barrier in vitro. Eur. J. Pharm. Biopharm. 2013, 84, 275-87.

(34) Nam, H. Y.; Kwon, S. M.; Chung, H.; Lee, S. Y.; Kwon, S. H.; Jeon, H.; Kim, Y.; Park, J. H.; Kim, J.; Her, S.; Oh, Y. K.; Kwon, I. C.; Kim, K.; Jeong, S. Y. Cellular uptake mechanism and intracellular fate of hydrophobically modified glycol chitosan nanoparticles. J. Controlled Release 2009, 135, 259-67.

(35) Pangarkar, C.; Dinh, A. T.; Mitragotri, S. Endocytic pathway rapidly delivers internalized molecules to lysosomes: an analysis of vesicle trafficking, clustering and mass transfer. J. Controlled Release 2012, 162, 76-83.

(36) Cheng, Z. J.; Singh, R. D.; Holicky, E. L.; Wheatley, C. L.; Marks, D. L.; Pagano, R. E. Co-regulation of caveolar and Cdc42dependent fluid phase endocytosis by phosphocaveolin-1. J. Biol. Chem. 2010, 285, 15119-25.

(37) Yue, Z.-G.; Wei, W.; Lv, P.-P.; Yue, H.; Wang, L.-Y.; Su, Z.-G.; Ma, G.-H. Surface Charge Affects Cellular Uptake and Intracellular Trafficking of Chitosan-Based Nanoparticles. Biomacromolecules 2011, 12, 2440-2446.

(38) Grant, B. D.; Donaldson, J. G. Pathways and mechanisms of endocytic recycling. Nat. Rev. Mol. Cell Biol. 2009, 10, 597-608.

(39) Desnick, R. J.; Schuchman, E. H. Enzyme replacement therapy for lysosomal diseases: lessons from 20 years of experience and remaining challenges. Annu. Rev. Genomics Hum. Genet. 2012, 13, $307-35$. 